\title{
Dextrose hydrodissection with ultrasound-guided as optional therapy in pronator teres syndrome: A case report
}

Yusak Mangara ${ }^{1 *}$, Tua Siahaan ${ }^{2}$

${ }^{1}$ Neurology Department, Faculty of Medicine, Universitas Pelita Harapan

${ }^{2}$ Neurology Department, Siloam Hospitals Lippo Village, Tangerang, Indonesia

*Corresponding Author: Yusak Mangara, Neurology Department, Faculty of Medicine, Universitas Pelita Harapan.

Received date: May 25, 2021: Accepted date: June 02, 2021: Published date: July 19, 2021

Citation: Yusak Mangara*, Tua Siahaan (2021) Dextrose hydrodissection with ultrasound-guided As optional therapy in pronator teres syndrome: A case report J. Clin Case Rep and Stu 2(3); DOI: 10.31579/2690-8808/078

Copyright: (C) 2021: Yusak Mangara. This is an open access article distributed under the Creative Commons Attribution License, which permits unrestricted use, distribution, and reproduction in any medium, provided the original work is properly cited.

\section{Abstract \\ Pronator teres syndrome (PTS) is a rare peripheral neuropathy of the proximal median nerve that occurs due to compression of the pronator teres muscle or its surrounding anatomical structures. Treatment options in cases of pronator syndrome include conservative therapy (oral, physical, intervention) to surgical therapy. Dextrose hydrodissection with ultrasound guided is an interventional therapy option in cases of peripheral neuropathy. This study presented a case of 50 year old female with chronic pronator teres syndrome who received ultrasound-guided 5\% dextrose hydrodissection therapy and experienced satisfactory resolution within 6 months of post injection evaluation. \\ Keywords: dextrose, hydrodissection, pronator teres syndrome, ultrasound-guided}

\section{Abbreviation}

AINS

ATP

CGRP

CTS

D5W

NRS

NS

NSAIDs

PRP

PTS

Quick-DASH

TRPV-1

\section{Introduction}

Pronator teres syndrome (PTS) or pronator syndrome is a syndrome of irritation of the proximal median nerve that occurs due to compression of the pronator teres muscle or the surrounding anatomical structures. [1][2][3][4] Pronator syndrome is included in a variety of cases of peripheral neuropathy of the median nerve that rarely occurs compared to the others, such as carpal tunnel syndrome (CTS) and anterior interosseous nerve syndrome (AINS). [2] In one study, the prevalence of pronator teres syndrome was mentioned in only $5 \%$ of all cases of median nerve neuropathy. This is in line with other studies, which reported that the distribution of cases of median compression was dominated by CTS (88.2\%) in the distal part followed by the proximal part (PTS and AINS) of $11.8 \%$. [3][5] PTS generally occurs in the fifth decade with female dominance. [3]

The clinical manifestation of PTS is pain in the anterior aspect of the forearm that is triggered by repetitive activities such as pronation or supination of the forearm and flexion of the fingers associated with occupation and habits. [3][6] This repetition results in hypertrophy of the muscles and the narrowing of the tunnel, where median nerve passes, leading to compression that will trigger neural inflammation leading to demyelination and perineural fibrosis. [2][3][6] Other symptoms found in PTS are paresthesia or numbness of the fingers 1,2,3 and lateral aspect of the $4^{\text {th }}$ finger and numbness of palm according to the branching of the median nerve. [3] Several studies have written that sensory disturbances in PTS vary widely and some cases resemble CTS. [2][3] In addition, PTS is also associated with local trauma conditions, tumors, and anatomical variants that allow mechanical compression of the pronator area. [2][4]

Hydrodissection therapy is an injection technique that involves injecting fluid (hydro) to release the nerve clamp (dissection) due to adhesions or obstruction of the surrounding tissue. With ultrasound guidance, this technique is considered safe and effective, especially in cases of nerve clamping, including PTS. [4][6][7] Several choices of fluids used in the hydrodissection technique include NS (Normal Saline), corticosteroids, 5\% dextrose (D5W), and PRP (Platelet-rich Plasma). [4][8] 5\% dextrose is very commonly used in clinical practice as there has not been found any significant side effects compared to other options. [9] However, until now the exact effectiveness of these medical properties has not been widely proven by various studies. [9][10] The study of Wu et al (2017) 
shows that dextrose hydrodissection intervention is safe and effective in treating cases of CTS. [11] This is in line with the meta-analysis study from Lin et al (2020) which concluded that D5W is the best therapy followed by PRP in the management of CTS. [12][13] However, the use of D5W hydrodissection in managing PTS has not been previously reported. This study describes a case with PTS that experienced significant improvement during the evaluation of 6 months after ultrasonography-guided D5W hydrodissection.

\section{Case}

A 50-year-old housewife complained pain and tingling in the proximal area of the forearm that radiates to four fingers (thumb to ring finger) on the left side since 6 months ago. Pain was described as burning and cramping, which limits daily activities, especially when gripping and lifting weights, with NRS (Numeric Rating Scale) 9 and Quick-DASH (Quick-Disabilities of Arm, Shoulder, and Hands) of 49.5\%. Patient has no history of trauma, tumors, and metabolic disorders such as Diabetes Mellitus and kidney disorders. Physical examination revealed a positive pronator compression test and tinel on the complained side. Motor strength of Medical Research Council scale on both arms were 5/5 and negative phalen test. Electromyography examination reveals the impression of a demyelinating-type median motor nerve neuropathy. Ultrasound shows fascicular swelling and epineural thickening. During the course of the disease, the patient had taken NSAIDs (non-steroidal anti-inflammatory drugs) and adjusted with lifestyle modifications but had never experienced any satisfactory resolution.

With the patient's consent, a long-axis perineural ultrasound-guided injection of the median nerve with 5\% dextrose is performed periodically ( $2 x, 2$ week intervals) by a certified neurologist. This procedure begins with positioning patient's elbow being extended). Then, the clinician uses a high-frequency linear transducer with a long-axis approach and points the needle from distal to proximal to perform hydrodissection. This needle placement aims to inject dextrose around the median nerve. (Figure. 1) Post-injection complications of infection or allergic reactions were denied. The NRS and Quick-DASH assessment after 3 months showed good results with NRS 0 and Quick-DASH of $8.25 \%$.

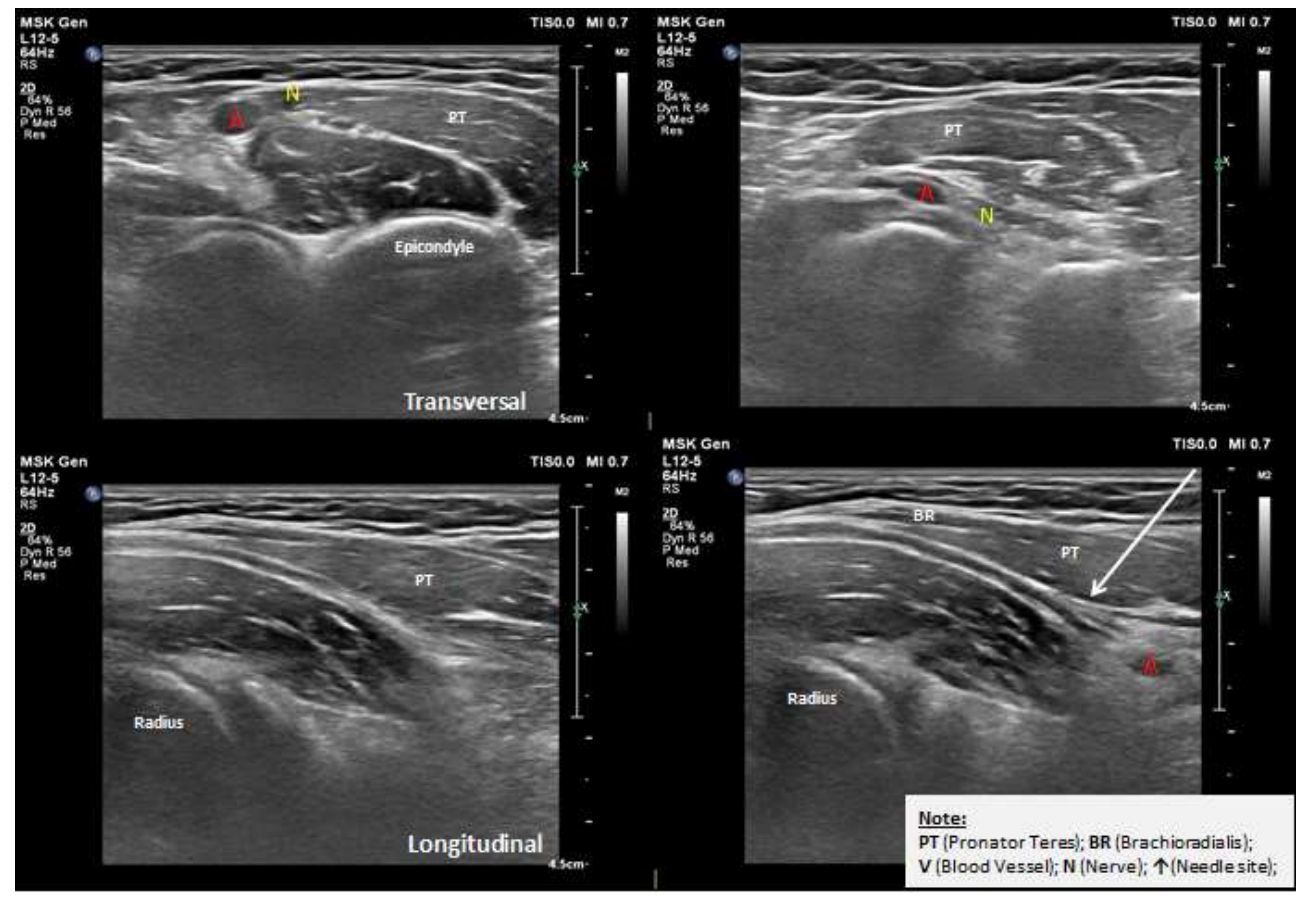

\section{Discussion}

The median nerve is supplied by the cervical rami of C6-T1 traversing the medial side elbow to the adjacent brachial artery. This nerve passes through the laceratus fibrosus and radial head, then passes between the radial and ulnar head of the pronator teres. Minimal compression of the nerve to the fascial layer in the pronator teres muscle area results in structural changes of the nerve. Pathophysiologically, light compression of the nerve area also compresses the small blood vessels outside the epineurium (nervi nervorum and vasa nervorum), thereby affecting the potential for stasis and accumulation of toxins resulting in neurogenic inflammation and producing neuropathic pain. [3] In this case, the patient complained of neuropathic described as burning and tingling sensation in the left forearm to the fingers 1-4 in accordance with the manifestations of compression of the median nerve. The PTS diagnosis is then confirmed through physical examination and supporting examinations.

Most cases of PTS are treated using conservative therapy for at least 6 weeks in the form of oral therapy (NSAIDs), physical therapy (immobilization, occupation, rehabilitation), to interventions (injection with local anesthetics to steroids) accompanied by lifestyle modifications. [2][6] If symptoms persist for more than 6 months with adequate conservative therapy, surgical therapy may be recommended as a last resort. [1][2][3] In this case, the patient had chronic severe pain and paresthesia affecting activity with NRS 9 and Quick-DASH 49.5\%. In addition, a history of oral NSAID medications and lifestyle modifications has been performed but has not shown satisfactory results. Therefore, clinicians should consider interventional hydrodissection therapy with D5W to obtain direct mechanical benefits, namely the release of compressed nerve entrapments resulting in restoration of function of nervi and vasa nervorum.

Hydrodissection of D5W is performed on the area around the peripheral nerves with ultrasound guidance to be able to release the peripheral nerves from the fascia covering them to give a decompression effect. Hydrodissection is performed between the brachial muscle and the pronator teres, not the pronator tunnel. The in-plane technique is recommended as a safer approach to dissecting the soft tissue in front of 
the needle, as well as completely removing the fascia until the nerve is rounded and the nerve is completely surrounded by the injection fluid.[611] After receiving the first ultrasound-guided D5W hydrodissection therapy, the patient experienced significant pain resolution with a decrease in the NRS score to 5 and a perceived reduction in paresthesia. Therefore, clinicians recommend periodic injections to improve sensory function in these patients.

Interventional therapy in the form of hydrodissection with ultrasound guidance has been widely applied in cases of peripheral neuropathy. [4][7][10] The latest study from Patel et al (2019) noted that 14 patients with PTS showed symptom improvement of $\geq 75 \%$ with steroid hydrodissection therapy for 3 months of evaluation. [6] Another study demonstrated that the use of D5W is more effective than other therapies because it has fewer side effects on blood vessels and tissues. [9][10][11][12] The use of lidocaine as a local anesthetic has a quick but temporary effect with systemic toxic effects that need attention. While corticosteroids, have long-term effects but there are effects of skin depigmentation, subcutaneous atrophy, and local infection. [10] However, apart from the choice of therapy, the accuracy of the injection technique also has an important role that cannot be ignored. [7] Those statements also supported the clinician's reason to choose D5W as optional therapy than others.

The effectiveness of D5W therapy on peripheral neuropathy is still being debated. In a previous study by Jensen et al (2008) the use of dextrose in prolotherapy was thought to induce local inflammatory stimulation. [14] However, several recent studies have argued that D5W actually plays a role in reducing neurogenic inflammatory processes by blocking the TRPV-1 receptor (Transient receptor Potential Vanilloid-type 1) found on peripheral nerves. [7][8][9][10] Apart from being a TRPV-1 antagonist, D5W also binds to presynaptic calcium channels and inhibits the release of substances P and CGRP (Calcitonin Genre Related Peptide) which also reduces neurogenic inflammation. It is suspected that neurotropic effects and growth factors facilitate nerve repair mechanisms and reduce pain. [8][9][11] In PTS, neuropathic pain occurs due to neural inflammation of the median nerve due to repetitive mechanical compression. [2][3][6] Chronic neuropathic pain significantly results in glycopenia of the surrounding nerves. This glycopenia results in decreased activity of ATP (Adenosine Triphosphate) and Na-K pump, leading to progressive nerve depolarization and hyperexcitability. Dextrose injection is considered to improve local glycopenia thereby reducing neuropathic pain that occurs. [8] Thus, the delivery of D5W to the perineural soft tissue (hydrodissection) is considered to be very helpful for nerve healing after decompression. Indirectly, the hydrodissection technique contributes to releasing adhesions to the surrounding tissue which allows the delivery of nerve impulses and reduces the risk of ischemia. [9] During the second injection, the patient experienced resolution of pain and loss of paresthesia. Evaluation for 6 months post injection was done, results showed NRS score of 0 and Quick-DASH 8.25\%. However, we recognize that a single case report cannot demonstrate the overall effectiveness of a treatment. Further studies with a larger sample will be needed to understand the effect of dextrose hydrodissection on PTS cases in the future.

\section{Conclusion}

This study represents the first case report representing ultrasound-guided dextrose hydrodissection that may be a therapeutic option for pronator teres syndrome. We hope that this study can become a trigger for the development of other studies with a larger sample and more in-depth discussion.

\section{Acknowledgement}

This report has none conflict of interest. The author would like to thank colleagues from Universitas Pelita Harapan and Siloam Hospital Lippo Village for supporting this manuscript.

\section{References}

1. Creteur V, Madani A, Sattari A, Bianchi S. (2017). Sonography of the Pronator Teres Normal and Pathologic Appearances. $J$ Ultrasound Med; 36: 2585-2597.

2. Dididze M, Tafti D, Sherman AI. Pronator Teres Syndrome. In: StatPearls [Internet]. Treasure Island (FL): StatPearls Publishing;

3. Bair MR, Gross MT, Cooke JR, Hill CH.(2016) Differential Diagnosis and Intervention of Proximal Median Nerve Entrapment: A Resident's Case Problem. Journal of Orthopaedic \& Sports Physical Therapy; 46(9): 800-808.

4. Verma S, Sakthivel S. (2016). A Case Report on Unilateral Accessory Humeral Head of Pronator Teres. Journal of Clinical and Diagnostic Research; 10(11); AD01-2.

5. Lee HJ, Kim I, Hong JT, Kim MS. (2014). Early Surgical Treatment of Pronator Teres Syndrome. J Korean Neurosurg Soc; 55(5): 296-269.

6. Patel M, Delzell PB. (2019) Ultrasound-Guided Perineural Injection for Pronator Syndrome Caused by Median Nerve Entrapment. J Ultrasound Med; 9999: 1-7.

7. Trescot A. (2016) Peripheral nerve entrapment, hydrodissection, and neural regenerative strategies. Techniques in Regional Anesthesia and Pain Management; 19(1): 1-9.

8. Lam KHS, Hung CY, Chiang YP, Onishi K, Ku DCJ, Clark TB, et al. (2020). Ultrasound-Guided Nerve Hydrodissection for Pain Management: Rationale, Methods, Current Literature, and Theoretical Mechanisms. Journal of Pain Research; 13: 19571968.

9. Chen SR, Shen YP, Ho TY, Chen LC, Wu YT. (2018). Ultrasound-guided perineural injection with dextrose for treatment of radial nerve palsy. Medicine (Baltimore); 97(23):e10978.

10. Chang K, Wu WT, Ozcakar L. (2020). Ultrasound imaging and guidance in peripheral nerve entrapment: hydrodissection highlighted. Pain Manag; 10(2):97-106. Doi: 10.2217/pmt-20190056.

11. Wu YT, Ho TY, Chou YC, et al. (2017). Six month efficacy of perineural dextrose for carpal tunnel syndrome: a prospective randomized double-blind controlled trial. Mayo Clin Proc; 92: 1179-1189.

12. Lin CP, Chang KV, Huang YK, Wu WT, Ozcakar L. (2020) Regenerative Injection including 5\% Dextrose and Platelet-Rich Plasma for Treatment of Carpal Tunnel Syndrome: A Systematic Review and Network Meta-Analysis. Pharmaceuticals; 13(49):117.

13. Ricci V, Becciolini M, Ozcakar L. (2020). Ultrasound-Guided Hydrodissection of the Median Nerve in Pronator Syndrome: Where is the Pronator Tunnel? J Ultrasound Med; 9999: 1-2.

14. Jensen KT, Rabago DP, Best TM, Patterson JJ, Vanderby R. (2008). Early Inflammatory Response of Knee Ligaments to Prolotherapy in Rat Model. J Orthop Res.; 26(6): 816-823. 